IGC- $-08 / 1-3$

\title{
Quantum nature of cosmological bounces
}

\author{
Martin Bojowald* \\ Institute for Gravitation and the Cosmos, \\ The Pennsylvania State University, \\ 104 Davey Lab, University Park, PA 16802, USA
}

\begin{abstract}
Several examples are known where quantum gravity effects resolve the classical big bang singularity by a bounce. The most detailed analysis has probably occurred for loop quantum cosmology of isotropic models sourced by a free, massless scalar. Once a bounce has been realized under fairly general conditions, the central questions are how strongly quantum it behaves, what influence quantum effects can have on its appearance, and what quantum space-time beyond the bounce may look like. This, then, has to be taken into account for effective equations which describe the evolution properly and can be used for further phenomenological investigations. Here, we provide the first analysis with interacting matter with new effective equations valid for weak self-interactions or small masses. They differ from the free scalar equations by crucial terms and have an important influence on the bounce and the space-time around it. Especially the role of squeezed states, which have often been overlooked in this context, is highlighted. The presence of a bounce is proven for uncorrelated states, but as squeezing is a dynamical property and may change in time, further work is required for a general conclusion.
\end{abstract}

\section{Introduction}

Classical cosmology as a description of the early universe is plagued by the big bang singularity. In this extreme high energy and high curvature regime quantum space-time is expected to take over the reigns from general relativity in a way which will hopefully cure the singularity problem. A common expectation is that the universe, instead of collapsing completely, will bounce at a minimum non-zero volume and thus connect to a well-defined pre-big bang state. Mediated by quantum gravity, such a picture would exploit the changes in forces and the dynamics which quantum physics should imply. If this can be realized, a central question remains: Do the quantum forces that have been summoned to fight the classical singularity leave space-time otherwise intact, or will space-time remain in a fluctuating turmoil long after the immediate danger of divergences has passed? Generally, strong quantum effects as they may be necessary to prevent the singularity lead to large

\footnotetext{
*e-mail address: bojowald@gravity.psu.edu
} 
fluctuations. Then, a genuine quantum space-time has to be dealt with, which may not be near a smooth bouncing effective picture.

Detailed examples have been provided by loop quantum cosmology [1], where homogeneous models are governed by a non-singular difference equation for quantum states [2, 3, 4, 5]. The general difference equation, however, does not provide direct insights into intuitive geometric properties around the classical singularity; in general the transition may not be semiclassical at all. But there is a class of models which are more easily accessible: isotropic models sourced solely by a free, massless scalar. Initial numerical calculations [6, 7] have suggested that the singularity is avoided by a surprisingly smooth bounce of a wave packet which remains sharply peaked and fluctuated before the bounce very nearly as it does afterwards. "Before" and "after" here refers to a global internal time which in these models is provided by the value of the scalar.

Upon closer examination, however, two new key features materialized:

- In a suitable factor ordering of the Hamiltonian constraint operator, a flat isotropic model with a free, massless scalar is a solvable system in which quantum backreaction does not occur [8]. This is the case even in the bounce phase of the loop quantization. Thus, quantum variables such as fluctuations or higher moments of a state do, although they are themselves dynamical and change in time, not influence the evolution of expectation values. It is this feature which makes the models highly controlled, but also very special. In quantum mechanics, for instance, this behavior is realized only for the harmonic oscillator. When interactions, or deviations from isotropy, are included quantum back-reaction does result and the evolution will become more quantum in the sense that it depends on how the quantum variables behave. Quantum correction terms due to coupling between expectation values and fluctuations would at first not be large for evolution starting with a semiclassical state, as it should be realized at large volume of a universe. However, states spread and deform from an initial Gaussian distribution. Accordingly, quantum variables generically grow in time and with them quantum corrections. This may take time, but can be significant in long-term evolution. Cosmology is a study of the system with the longest possible evolution times, and especially regarding the big bang singularity the correct question to ask is: What is the behavior of a universe at small volume, described by a generic quantum state as it has evolved from a semiclassical state at large volume? This question cannot be answered if the behavior of the quantum system is known only for semiclassical states and rather short evolution times. For all we know, the universe near the big bang can be in a highly quantum state. Thus, quantum back-reaction is a serious player in this regime, and one has to understand how it may affect the bounce of the free solvable model.

- Even within the solvable model, fluctuations before and after the bounce can be very different from each other [9, 10].1] This asymmetry is not a consequence of quantum

\footnotetext{
${ }^{1}$ Some of the recent literature on this topic has witnessed a certain amount of reluctance to accept this property. For instance, it has been claimed in [11] that fluctuations of a state which is semiclassical at
} 
back-reaction but of squeezing. As the precise state is unknown, there is no strong control on how it may be squeezed even when it is semiclassical. Accordingly, no strong control on fluctuations during and before the big bang exists. Still, for any such state there is a bounce in this free model provided that the state is semiclassical at one time.

The formulation in [8] as a solvable model allows the derivation of precise effective equations in this case, which easily shed light on the evolution of a quantum space-time and its properties. If the model is perturbed by including a small matter potential, perturbative effective equations can still be derived in an expansion [12]. In this paper, we use these equations to show that the bounce is in fact affected by interactions and determine how this is related to the possible squeezing of the state. Our analysis is thus based on a combination of the two key results which are realized in isotropic, free scalar models. We provide a new effective equation which, unlike previous examples, is valid for a massive or self-interacting scalar. It contains additional correction terms which are especially important near a wouldbe bounce.

\section{Loop quantum cosmology}

In loop quantum cosmology, the basic pair of canonical variables is formed by the square $p=a^{2}$ of the scale factor $a$ and extrinsic curvature, i.e. the time derivative $c=\gamma \dot{a}$. Their Poisson bracket is $\{c, p\}=8 \pi \gamma G / 3$. These are isotropic reductions of a densitized triad $E_{i}^{a}=p \delta_{i}^{a}$ and the Ashtekar connection $A_{a}^{i}=c \delta_{a}^{i}$ and enter the basic operators of a loop quantization. Although $p$ can take both signs, signifying the two possible orientations of the triad, we will mainly restrict attention to positive values.

While $p$, just as the densitized triad of the full theory, can readily be quantized through flux operators, there is no operator for the connection components. Instead, holonomies of the connection are represented in a well-defined way, which are obtained after integrating the connection along curves and exponentiating the result. In an isotropic model, it is not obvious what the length of the curve used in the integration should be, thus giving rise to quantization ambiguities which can only be resolved in a detailed reduction of the isotropic model from the full quantum theory. (See [13, 14, 15, 16, 17, 18, 19] for work in this direction, which remains incomplete.) What length 2 is used should depend on the underlying state and its scale of discreteness, which in general should be time dependent.

large volume have to be very nearly symmetric around the bounce. What was actually derived there was an upper bound for the difference before and after the bounce of fluctuations of volume relative to the total volume. However, the discussion ignored the size of these relative fluctuations and overlooked the fact that each of the terms in the difference, not just the difference, is smaller than the upper bound provided. Thus, the inequality does not at all restrict the asymmetry. One can easily see that the numerical example provided in [11] allows for ratios of relative fluctuations before and after the bounce as large as $10^{28}$. Judging from this estimate alone, a state before the big bang may be highly non-semiclassical even if it becomes very semiclassical after the big bang. The detailed bounds provided in [10] are much sharper, but still leave room for asymmetric fluctuations.

${ }^{2}$ The notion of length here only refers to coordinates and so it may seem that it is not covariant. 
Thus, also the length factor $\mu$ of a holonomy $\exp (i \mu c)$ in an isotropic model could be a function of time, or rather of the spatial geometry since this determines the evolving scales.

A quantum theory with a time dependent number of degrees of freedom is difficult to formulate and analyze [20, 21, 22, 23, 24, but key properties can be included in the description of an isotropic model. Instead of using a fixed $\mu \in \mathbb{R}$, we formulate the dynamics with holonomies of the form $\exp (i f(p) c)$ where $f(p)$ takes into account a possible dependence of the discreteness scale on the total size. When quantized, the exponential of the momentum $c$ becomes a shift operator in $p$, rather than a derivative operator. For $f(p)=$ const, the basic triad variable $p$ would be equidistantly spaced when acting with powers of holonomies, but not for a $p$-dependence of $f(p)$. Of special interest are functions $f(p)=f_{0} p^{x}$ of power-law form, which can arise from loop quantum gravity for $-1 / 2<x<0$ [17]. Several independent phenomenological and stability arguments prefer a value near the lower bound $x \approx-1 / 2[25,26,27,28,29,30]$. For arbitrary $x$, a canonical pair is formed by the new variables $(f(p) c, V)$ with

$$
V:=\frac{3 p}{8 \pi \gamma G(1-x) f(p)}=\frac{3 a^{2(1-x)}}{8 \pi \gamma G(1-x) f_{0}} \quad \text { such that } \quad\left\{f_{0} p^{x} c, V\right\}=1 .
$$

(We have $V$ proportional to the spatial volume for the power-law case $f(p) \propto p^{-1 / 2}$. In this case, the number of vertices in a graph would grow in a way proportional to spatial volume: $\mathcal{N}(a)=f\left(a^{2}\right)^{-3} \propto a^{3}$. ) This shows that for a given $x, p / f(p)$ is equidistantly spaced upon action of $\exp ($ if $(p) c)$.

In loop quantum cosmology, we have to quantize the classical Friedmann equation

$$
\left(\frac{\dot{a}}{a}\right)^{2}=\frac{8 \pi G}{3} \frac{H_{\text {matter }}(a)}{a^{3}}=\frac{8 \pi G}{3} \rho
$$

where $H_{\text {matter }}$ is the Hamiltonian of matter and $\rho$ its energy density. This is done via the Hamiltonian constraint, which is obtained by multiplying the Friedmann equation with $a^{3}$. For a free, massless scalar, we have the matter Hamiltonian $H_{\text {matter }}=\frac{1}{2} a^{-3} p_{\phi}^{2}$ with the scalar momentum $p_{\phi}$. The gravitational part requires the use of $c$, which in loop quantum

However, no such problems or even issues with potential gauge artifacts arise as one can see upon closer examination [17. To see this, we have to refine our definition of the basic variables as $p=V_{0}^{2 / 3} a^{2}$ and $c=V_{0}^{1 / 3} \gamma \dot{a}$ where $V_{0}$ is the coordinate volume of a region chosen for the spatial integration of the symplectic structure and the Hamiltonian [4]. Holonomies then take the form $\exp \left(i \ell_{0} V_{0}^{-1 / 3} c\right)$ where $\ell_{0}$ is the coordinate length of an edge as referred to above. This immediately shows that the expression is not coordinate dependent. It also does not depend on the choice of $V_{0}$ since this dependence cancels between $c$ and $V_{0}^{-1 / 3}$. Instead of referring to a coordinate quantity, the length factor can be interpreted as $\mathcal{N}^{-1 / 3}$ where $\mathcal{N}=V_{0} / \ell_{0}^{3}$ is roughly the number of vertices of an underlying spin network state in the volume $V_{0}$. This immediately shows the dynamical aspect of a pre-factor $f(p)$ in $\exp (i f(p) c)$, which includes the factor $V_{0}$, if the graph is being refined during evolution. In this way, $\mathcal{N}(p)$ will be the number of vertices of the state at volume $p^{3 / 2}$. In this context, notice that a solution to the Hamiltonian constraint equation in general is a superposition of many states associated with different graphs. The function $\mathcal{N}(V)$ refers to this relationally as the number of vertices of graphs in a decomposition as eigenstates of the volume operator. This indicates how $f(p)$ refers to internal time evolution in a constrained theory. 
cosmology can only be formulated by means of holonomies which are periodic functions of their arguments. There is no exact correspondence, but all we need is a well-defined operator which has (2) as its low-curvature limit. Many examples for such operators exist, the simplest choice being of the form

$$
\left.\frac{3}{8 \pi \gamma^{2} G}\left(f(p)^{-2} \widehat{\sin ^{2}(f}(p) c\right) \sqrt{p}\right) \psi(p, \phi)=-\frac{1}{2} \hbar^{2} \widehat{p^{-3 / 2}} \frac{\partial^{2}}{\partial \phi^{2}} \psi(p, \phi) .
$$

(See e.g. [31] for a discussion of some ambiguities in this case.) We have not yet decided on the factor ordering between $p$ and $c$ on the left hand side, and also the right hand side is not fully defined as written because $p^{-3 / 2}$ requires an inverse, which does not exist for the operator $\hat{p}$ with its discrete spectrum containing zero in loop quantum cosmology. Its quantization thus has to be more indirect, which can be done [32] following general constructions of the full theory [33. (Also here, ambiguities arise [34, 35] without changing the qualitative picture.)

\subsection{Difference equation}

Basic operators of the quantization are the flux operator $\hat{p}|\mu\rangle=\frac{4}{3} \pi \gamma \ell_{\mathrm{P}}^{2} \mu|\mu\rangle$ with the Planck length $\ell_{\mathrm{P}}=\sqrt{G \hbar}$, whose orthonormal eigenstates $|\mu\rangle$ with $\mu \in \mathbb{R}$ we are using, and the holonomy operator $\exp \left(i \mu^{\prime} c / 2\right)|\mu\rangle=\left|\mu+\mu^{\prime}\right\rangle$. By expressing the Friedmann equation in terms of holonomies, which then become finite shift operators, one thus obtains a difference equation for wave functions [36, 3].

There are several ingredients for an explicit expression of the difference equation: First, using holonomies for a given refinement scheme we refer to shift operators $\exp ($ if $(p) c / 2)|\mu\rangle \sim$ $|\mu+F(\mu)\rangle$ of a $\mu$-dependent step-size, where $F(\mu):=f\left(\frac{4}{3} \pi \gamma \ell_{\mathrm{P}}^{2} \mu\right)$. (This action is not precisely written yet due to factor ordering which is discussed below.) The factor $\sqrt{p}$ in (3) could be quantized directly via $\hat{p}$, but is usually written in a way closer to what one has in the full theory. The reason is that in an inhomogeneous expression one would instead have a factor $E_{i}^{a} E_{j}^{b} / \sqrt{\left|\operatorname{det}\left(E_{k}^{c}\right)\right|}$ which, due to the inverse of the determinant, cannot be quantized directly. Following the same steps in an isotropic model, one is led to a quantization of $\sqrt{p}$ of the form if $(p)^{-1} \exp (i f(p) c)\left[\exp (-i f(p) c),|\hat{p}|^{3 / 2}\right]$ which also happens to be diagonalized by the triad eigenstates $|\mu\rangle$. (Note, however, that the diagonalization property does not hold in the full theory [37, 38].) Finally, the factors $f(p)^{-2}$ and $p^{-3 / 2}$ have to be turned into operators.

An explicit realization faces two main issues: (i) the non-equidistancy of step-sizes and (ii) the factor ordering. The ordering between $\sqrt{q}$ and $f(p)^{-2} \sin ^{2}(f(p) c)$ can be mimicked from the full theory [39, 40], which leads one to order the quantization of $\sqrt{p}$ to the right to obtain the basic Hamiltonian constraint operator $\hat{H}$ which may in a second step be ordered symmetrically as $\frac{1}{2}\left(\hat{H}+\hat{H}^{\dagger}\right)$. However, the factors of $f(p)$ model the refinement behavior of an underlying lattice state, and thus do not occur in the full theory where the lattice refinement is automatically realized by elementary holonomies. Thus, the ordering in isotropic models is not strictly defined, and one cannot always use guidance from the full 
theory. In applications, one should therefore use only properties which are insensitive to the factor ordering. This is also useful for the first issue: the non-equidistancy of the resulting difference equations. As shown in [26], one can always transform a difference equation of isotropic models to equidistant form, up to changing the factor ordering. Thus, one can use preferred orderings in which an equidistant difference equation results which is much easier to analyze. (See [41] for a numerical procedure to analyze non-equidistant difference equations directly.)

Specifically, we obtain a Hamiltonian constraint operator

$\left(\hat{H}-\hat{H}_{\text {matter }}\right)|\mu\rangle=\frac{1}{32 \sqrt{6} \pi \gamma^{3 / 2} G}\left(|\mu+F(\mu)|^{3 / 2}-|\mu-F(\mu)|^{3 / 2}\right) F(\mu)^{-3}(|\mu+4 F(\mu)\rangle-2|\mu\rangle+|\mu-4 F(\mu)\rangle)$

by following the steps detailed in [3, 1]. A physical state $|\psi\rangle=\sum_{\mu} \psi_{\mu}(\phi)|\mu\rangle$ has to satisfy $\hat{H}|\psi\rangle=0$, which for its coefficients translates into the difference equation

$$
\begin{aligned}
& \left|\Delta_{F}(\mu+4 F(\mu))\right| F(\mu+4 F(\mu))^{-3} \psi_{\mu+4 F(\mu)}(\phi)-2\left|\Delta_{F}(\mu)\right| F(\mu)^{-3} \psi_{\mu}(\phi) \\
& +\left|\Delta_{F}(\mu-4 F(\mu))\right| F(\mu-4 F(\mu))^{-3} \psi_{\mu-4 F(\mu)}(\phi)=-64 \sqrt{6} \pi \gamma^{3 / 2} G \hat{H}_{\text {matter }}(\mu) \psi_{\mu}(\phi)(5)
\end{aligned}
$$

where $\Delta_{F}(\mu):=|\mu+F(\mu)|^{3 / 2}-|\mu-F(\mu)|^{3 / 2}$. (The Wheeler-DeWitt equation in a particular factor ordering is reproduced in the continuum or large volume limit where $\mu \gg F(\mu)$ at large $\mu$ 42.)

While such a difference equation of varying step-size $F(\mu)$ is difficult to solve and analyze, it may be transformed to an equidistant equation up to factor ordering. For this, we use $\psi_{\mu+F(\mu)}=\tilde{\psi}_{\tilde{\mu}+1}-\frac{1}{2} F^{\prime}(\mu) \tilde{\psi}^{\prime}+\cdots$ where omitted terms are higher derivatives of $\tilde{\psi}_{\tilde{\mu}}:=\psi_{\mu(\tilde{\mu})}$ by $\tilde{\mu}(\mu):=\int^{\mu} \mathrm{d} \nu / F(\nu)$. All derivative terms can be shown to be corrections of higher order in $\hbar$, such that one may use equidistant shift operators in $\tilde{\mu}$ up to quantum corrections which one can absorb in the ordering choice [26]. For specific refinement models of power-law form $F(\mu) \propto \mu^{x}$, we have an equidistant equation in the variable $\tilde{\mu} \propto \mu^{1-x}$, which would be proportional to volume $\mu^{3 / 2}$ for $x=-1 / 2$.

In the region around $\mu \sim 0$, which is a strong quantum region, the equidistant difference equation may differ significantly from the original, non-equidistant one. Moreover, at those small volumes it is difficult to find a specific form of refinement function $F(\mu)$ which could reliably capture refinements of small lattices. A power law $f(p) \propto p^{x}$ with $x<0$ can clearly not be used there. It is thus fortunate that the general difference equation (5) can be used to conclude general singularity avoidance: In a solution scheme one can simply step over the values of the wave function at $\mu=0$ where the classical singularity would be [2, 5]. The arguments given in [2] for constant step-size are unchanged for an equation of the form (5) provided that $F(0) \neq 0$ as it is required for a well-defined difference equation. Thus, quantum evolution of the wave function continues beyond the classical singularity, which as this general statement has been shown also for anisotropic [43, 44] and spherically symmetric models [45]. 


\subsection{Harmonic cosmology}

We are interested in quantum corrections as they arise when an initial semiclassical state, which solves a difference equation such as (5) becomes more quantum. We can therefore make several approximations by ignoring quantum effects which are not expected to be strong or which would not change the qualitative effect of quantum back-reaction. An example for the first effect is the inverse $\widehat{\left\langle a^{-3}\right\rangle}$, which differs from $a^{-3}$ at small scales. As such scales are not reached in what we consider here 3 we can assume $\hat{a}^{3} \widehat{a^{-3}} \approx 1$. An example for the second effect is factor ordering choices, which will change the precise values of quantum correction terms but not their qualitative behavior. Thus, our analysis is not sensitive to the fact that the ordering of the Hamiltonian constraint in loop quantum cosmology is not fully fixed. Taking a square root in (3), we thus obtain a Schrödinger equation

$$
\left.-\hat{p}_{\phi} \psi(p, \phi)=i \hbar \frac{\partial}{\partial \phi} \psi(p, \phi)= \pm \sqrt{\frac{3}{4 \pi G}} \gamma^{-1} \mid f(p)^{-1} \widehat{\sin (f}(p) c\right) p \mid \psi(p, \phi)=: \pm \hat{H} \psi(p, \phi)
$$

whose main trace of the loop quantization is the occurrence of the sine 4

There is a specific factor ordering for which the system becomes exactly solvable [8]. Since there are no general restrictions on the ordering, we choose this one for our analysis. Again, we emphasize that conclusions drawn are reliable only if they are insensitive to the factor ordering. For this, we use our canonical variables $(f(p) c, V)$ and introduce $J:=V \exp (i f(p) c)$ which depends on $c$ only in a form allowed by the loop quantization. (Using $V$ and $J$ as basic variables implies that the phase-space appears as a cone since the mapping from $(p, c)$ to $(V, J)$ is not one-to-one at $V=0$. But outside $V=0$, which is our main interest, we obtain a correct description.) For operators, ordering $\hat{J}$ as indicated in its definition, we have the commutation relations

$$
[\hat{V}, \hat{J}]=\hbar \hat{J} \quad, \quad\left[\hat{V}, \hat{J}^{\dagger}\right]=-\hbar \hat{J}^{\dagger} \quad, \quad\left[\hat{J}, \hat{J}^{\dagger}\right]=-2 \hbar \hat{V}-\hbar^{2}
$$

of an $\operatorname{sl}(2, \mathbb{R})$ algebra. Most importantly, the Hamiltonian is a linear combination of these basic operators, $\hat{H}=-\frac{1}{2} i\left(\hat{J}-\hat{J}^{\dagger}\right)$, which guarantees solvability, i.e. the decoupling of

\footnotetext{
${ }^{3}$ Inverse scale factor corrections are not dominant in isotropic models for large matter content (although they can be larger than sometimes assumed; see the appendix of [26]). They do become important in inhomogeneous situations where local energies smaller than the total matter must be used.

${ }^{4}$ The harmless-looking absolute value on the right hand side would be difficult to compute explicitly for operators, which would make them non-local in specific $p$ - or $c$-representations. Here, however, this will not present a problem at all: We simply need to require an initial state to have a decomposition in $\hat{H}$-eigenstates where only states with positive eigenvalues occur. Since $\hat{H}$ is preserved, this property will continue to hold for evolved states. Imposing the absolute value is thus not an issue of evolution, but of initial states which can be much more easily dealt with (for $\Delta p_{\phi} \ll\left|p_{\phi}\right|$ which we are going to use). One may also worry that superpositions are not allowed of the right kind: positive- and negative frequency (i.e. $\hat{H}$-eigenstates with positive and negative eigenvalue, respectively) instead of left- and right-moving. We do not allow superpositions of the first kind as per the condition on initial states. We do, however, allow superpositions of left- and right-moving states (corresponding to the \pm -choice in (6)). Note, however, that we are going to compute primarily expectation values and fluctuations, which are of interest only for individual wave packets in a superposition, not for the total superposition.
} 
quantum variables from expectation values. (We are ignoring factors of $4 \pi G / 3$ and $1-x$ which would simply rescale $\phi$ in our solutions. They can be reinstated simply by multiplying $H$ with $2 \sqrt{4 \pi G / 3}(1-x)$, which we will do in our effective Friedmann equation.)

We are still dealing with a difference equation of the type (5), but in a specific ordering which will allow us to get access to interesting properties of its solutions much more easily via expectation values and fluctuations. The specific difference equation reads

$$
-\hat{p}_{\phi} \psi=i \hbar \frac{\partial \psi}{\partial \phi}=\hat{H} \psi=\frac{1}{2 i}\left(\tilde{\mu} \psi_{\tilde{\mu}-2}-(\tilde{\mu}+2) \psi_{\tilde{\mu}+2}\right)
$$

which is a first-order version of

$$
\hbar^{2} \frac{\partial^{2} \psi}{\partial \phi^{2}}=\frac{1}{4}\left(\tilde{\mu}(\tilde{\mu}-2) \psi_{\tilde{\mu}-4}-\left(\tilde{\mu}^{2}+(\tilde{\mu}+2)^{2}\right) \psi_{\tilde{\mu}}+(\tilde{\mu}+2)(\tilde{\mu}+4) \psi_{\tilde{\mu}+4}\right) .
$$

Rather than solving for wave functions and then computing their expectation values and fluctuations of $\hat{V}$ and $\hat{J}$, we compute those quantities directly. Expectation values satisfy equations of motion

$$
\frac{\mathrm{d}}{\mathrm{d} \phi}\langle\hat{V}\rangle=\frac{\langle[\hat{V}, \hat{H}]\rangle}{i \hbar}=-\frac{1}{2}\left(\langle\hat{J}\rangle+\left\langle\hat{J}^{\dagger}\right\rangle\right) \quad, \quad \frac{\mathrm{d}}{\mathrm{d} \phi}\langle\hat{J}\rangle=\frac{\langle[\hat{J}, \hat{H}]\rangle}{i \hbar}=-\langle\hat{V}\rangle-\frac{1}{2} \hbar=\frac{\mathrm{d}}{\mathrm{d} \phi}\left\langle\hat{J}^{\dagger}\right\rangle
$$

which are not coupled to fluctuations or higher moments and can be solved easily. For arbitrary states, we have

$$
\begin{aligned}
\langle\hat{V}\rangle(\phi) & =\frac{1}{2}\left(A e^{-\phi}+B e^{\phi}\right)-\frac{1}{2} \hbar \\
\langle\hat{J}\rangle(\phi) & =\frac{1}{2}\left(A e^{-\phi}-B e^{\phi}\right)+i H
\end{aligned}
$$

with two constants of integration $A$ and $B$ and $H=\langle\hat{H}\rangle$.

We have to impose reality conditions to ensure that the variable $c$ appearing in the complex $J$ is real. Classically, this is equivalent to $J \bar{J}=V^{2}$, which also has to apply for operators: $\hat{J} \hat{J}^{\dagger}=\hat{V}^{2}$. (But note $\hat{J}^{\dagger} \hat{J} \neq \hat{V}^{2}$.) Upon taking an expectation value, this condition relates expectation values and second order quantum fluctuations:

$$
|\langle\hat{J}\rangle|^{2}-\left(\langle\hat{V}\rangle+\frac{1}{2} \hbar\right)^{2}=(\Delta V)^{2}-C_{J \bar{J}}+\frac{1}{4} \hbar^{2}
$$

where the $V$-fluctuation and $J$ - $\bar{J}_{\text {-covariance }}$

$$
(\Delta V)^{2}=\left\langle\hat{V}^{2}\right\rangle-\langle\hat{V}\rangle^{2} \quad \text { and } \quad C_{J \bar{J}}=\frac{1}{2}\left\langle\hat{J} \hat{J}^{\dagger}+\hat{J}^{\dagger} \hat{J}\right\rangle-\langle\hat{J}\rangle\left\langle\hat{J}^{\dagger}\right\rangle
$$

appear. If a state is semiclassical at least once, $(\Delta V)^{2}-C_{J \bar{J}}$ is initially of the order $\hbar$ and remains so because it is preserved in time [46]. (See also the explicit solutions (16) and (21) below.) Thus, inserting the solutions (9) and (10) yields $A B=H^{2}+O(\hbar)$. This 
implies that $A$ and $B$ must have the same sign, and we can define $A / B=: e^{2 \delta}$ to write the solution as $\langle\hat{V}\rangle(\phi)=H \cosh (\phi-\delta)$ (ignoring the small contribution $-\frac{1}{2} \hbar$ ). Thus, there is no singularity of vanishing volume but instead a bounce at $\phi=\delta$. Large $H$, i.e. much matter in the universe, ensures that other quantum corrections not included here do not destroy the bounce. For instance, corrections in $\widehat{a^{-3}}$ or from the factor ordering are not relevant in this case. For small $H$, however, there is no guarantee for a semiclassical bounce as $(\Delta V)^{2}-C_{J \bar{J}}$ could possibly compensate the positive $H^{2}$ and make $A B$ negative such that $\langle\hat{V}\rangle(\phi)$ would be sinh-like. (There may be quantizations in which all states bounce irrespective of whether they are semiclassical even at only one time. But this would depend sensitively on factor ordering choices, which we do not consider a reliable property.)

\subsubsection{Free effective Friedmann equation}

As with any linear system, one can easily derive precise effective equations for the harmonic model of cosmology. They have the advantage of being more intuitive to interpret and thus give a more direct handle on geometrical aspects which would be difficult to decipher from a wave function. To derive effective equations [47, 48], one first defines the quantum Hamiltonian as the expectation value $H_{Q}=\langle\hat{H}\rangle$ in a general state, parametrized by its expectation values and moments. The quantum Hamiltonian is thus a function of the expectation values and all infinitely many moments, determining their equations of motion via Poisson brackets as they follow from quantum commutators. However, all infinitely many equations of motion for the quantum variables are in general coupled to each other and to the expectation values, which usually makes these equations impossible to solve. One thus has to look for suitable approximations based on a truncation of the coupled system to a finite size of equations and variables. The truncated equations are the effective equations, which describe properties of the quantum system in regimes where the approximations used are valid.

In a linear system such as our solvable model for a quantum space-time, no truncation is required as the variables automatically decouple into sets of finitely many equations. Thus, the quantum Hamiltonian provides precise effective equations which in our case follow from $H_{\text {eff }}=\langle\hat{H}\rangle=-\frac{1}{2} i(J-\bar{J})$, only depending on expectation values. (In the context of the effective Friedmann equation we drop brackets denoting expectation values.) For expectation values, the equations (8) provided before are thus precise effective equations. It is sometimes useful to formulate them as evolution equations in proper time rather than internal time $\phi$, as this can then readily be compared with the classical Friedmann equation. To derive this, we use $H_{\text {eff }}=-i \sqrt{4 \pi G / 3}(1-x)(J-\bar{J})=p_{\phi}$, where we now have reinstated the numerical factors, and write the equation of motion

$$
\frac{\mathrm{d} V}{\mathrm{~d} \phi}=-\sqrt{\frac{4 \pi G}{3}}(1-x)(J+\bar{J})=\mp 2 \sqrt{\frac{4 \pi G}{3}}(1-x) V \sqrt{1-\frac{3 p_{\phi}^{2}}{16 \pi G(1-x)^{2} V^{2}}+\sigma} .
$$

Here, we have eliminated $J+\bar{J}$ using the reality condition (11) which implies

$$
\frac{1}{4}\left((J+\bar{J})^{2}+(i(J-\bar{J}))^{2}\right)=J \bar{J}=(V+\hbar / 2)^{2}+(\Delta V)^{2}-G^{J \bar{J}}+\frac{\hbar^{2}}{4}
$$


and thus

$$
\frac{J+\bar{J}}{2 V+\hbar}= \pm \sqrt{1-\left(\frac{J-\bar{J}}{i(2 V+\hbar)}\right)^{2}+\sigma}
$$

with a small $\sigma:=\left((\Delta V)^{2}-G^{J \bar{J}}+\hbar^{2} / 4\right) /(V+\hbar / 2)^{2}$ as a relative quantum variables. (This relation reflects the identity $\cos (f(p) c)^{2}+\sin (f(p) c)^{2}=1$, corrected by factor ordering terms which arise from the quantized $J$. The contribution $\frac{1}{2} \hbar$ to $V$ can safely be ignored for our purposes. Notice that the derivation of $\sigma$ shows that its form depends on factor ordering choices in the Hamiltonian and basic variables.)

To reformulate this as a Friedmann equation, we use the expression for $V$ in terms of $a$ of (1) for a refinement function $f(p)=f_{0} p^{x}$. Proper time then enters through 5 $\dot{\phi}=\left\{\phi, H_{\text {matter }}\right\}=a^{-3} p_{\phi}$, which implies the corrected Friedmann equation

$$
\left(\frac{\dot{a}}{a}\right)^{2}=\left(\frac{1}{2(1-x)}\right)^{2}\left(\frac{\dot{V}}{V}\right)^{2}=\frac{4 \pi G}{3} \frac{p_{\phi}^{2}}{a^{6}}\left(1-\frac{4 \pi G}{3} \gamma^{2} f_{0}^{2} a^{2+4 x} \frac{p_{\phi}^{2}}{a^{6}}\right)
$$

for a free, massless scalar. As one can easily see, the quantum correction can simply be formulated as a term quadratic in the energy density of the free scalar [49] (see also [50, 51, 31]). The bounce manifests itself since $\dot{a}=0$ is possible if $\rho_{\text {free }}=\frac{1}{2} p_{\phi}^{2} / a^{6}=\rho_{\text {crit }}=$ $3 a^{-2-4 x} / 8 \pi G f_{0}^{2} \gamma^{2}$. For $x=-1 / 2$ and $f_{0} \sim \ell_{\mathrm{P}}$, this is a Planckian energy density, but can be smalle1 6 for larger $f_{0}$ or $x>-1 / 2$.

For a general interpretation, one should note, however, that higher powers of $\rho$ are not the primary correction in loop quantum cosmology. The reason for the new term is the higher curvature corrections from the presence of the sine in the effective Hamiltonian, which in this model can be reformulated as a simple correction of the energy dependence. This proves the correctness of the effective Friedmann equation, as realized in [8], but only for this specific matter content. While it is tempting to use (14) for all matter contents, just replacing $p_{\phi}^{2} / 2 a^{6}$ with $\rho$, this would overlook corrections arising from quantum backreaction which must be present in general. We will come back to these corrections in the next section, where we derive correct effective equations in the presence of self-interactions.

\footnotetext{
${ }^{5}$ As we are not including quantum corrections in the matter Hamiltonian, this relation is identical to the classical one.

${ }^{6}$ Moreover, the critical density would be $a$-dependent for $x \neq-1 / 2$. In this case, it is not just the matter density which plays a role for the bounce but also the underlying quantum gravitational state and its refinement. There is no a priori reason why only the matter density should play a role for the bounce. In fact, it is the spatial discreteness of the quantum representation which implies a repulsive force, and thus the quantum gravity state is the primary reason for the bounce. In the language of refinement models, including the coordinate volume $V_{0}$, we have $f_{0}^{2} a^{2+4 x}=f(p)^{2} p \sim \mathcal{N}^{-2 / 3} p=\left(a^{3} / \mathcal{N}\right)^{2 / 3}$ which is the value of an elementary flux, or roughly the area $L^{2}$ of an elementary lattice plaquette. In terms of $L^{2}$, we have the critical density $\rho_{\text {crit }}=3 / 8 \pi G \gamma^{2} L^{2}$ for any $x$, which clearly shows that the critical density is determined by the underlying lattice. For $x=-1 / 2, L$ happens to be independent of $a$, and thus the critical density is a constant. For $x \neq-1 / 2$, on the other hand, the elementary flux depends on the total spatial size in a way determined by the lattice refinement, thus also making the critical density $a$-dependent. Requiring $L$ to be constant and of the order $\ell_{\mathrm{P}}$ implies that $x=-1 / 2$ and makes the critical density Planckian, which produces the parameter choices of [7]. However, this requirement is not necessary.
} 


\subsubsection{Quantum variables}

The solution procedure can be repeated for higher moments of a state, for which the same effective Hamiltonian $H_{\text {eff }}=-\frac{1}{2} i\left(\langle\hat{J}\rangle-\left\langle\hat{J}^{\dagger}\right\rangle\right)$ provides equations of motion. Hamiltonian equations of motion, for instance for volume fluctuations $(\Delta V)^{2}=\left\langle\hat{V}^{2}\right\rangle-\langle\hat{V}\rangle^{2}$, are then derived by means of Poisson brackets which follow from expectation values of commutators: using $\left\{\left\langle\hat{V}^{2}\right\rangle,\langle\hat{J}\rangle\right\}=\left\langle\left[\hat{V}^{2}, \hat{J}\right]\right\rangle / i \hbar=-i\langle\hat{V} \hat{J}+\hat{J} \hat{V}\rangle$ and $\left\{\langle\hat{V}\rangle^{2},\langle\hat{J}\rangle\right\}=2\langle\hat{V}\rangle\langle[\hat{V}, \hat{J}]\rangle / i \hbar=$ $-2 i\langle\hat{V}\rangle\langle\hat{J}\rangle$ we have $\left\{(\Delta V)^{2},\langle\hat{J}\rangle\right\}=-2 i C_{V J}$. Other Poisson brackets can be derived analogously (see the App. $\mathrm{A}$ and [12] for explicit expressions), which results in equations of motion

$$
\begin{aligned}
\frac{\mathrm{d}}{\mathrm{d} \phi}(\Delta V)^{2} & =-C_{V J}-C_{V \bar{J}} \quad, \quad \frac{\mathrm{d}}{\mathrm{d} \phi}(\Delta J)^{2}=-2 C_{V J} \quad, \quad \frac{\mathrm{d}}{\mathrm{d} \phi}(\Delta \bar{J})^{2}=-2 C_{V \bar{J}} \\
\frac{\mathrm{d}}{\mathrm{d} \phi} C_{V J} & =-\frac{1}{2}(\Delta J)^{2}-\frac{1}{2} C_{J \bar{J}}-(\Delta V)^{2} \quad, \quad \frac{\mathrm{d}}{\mathrm{d} \phi} C_{V \bar{J}}=-\frac{1}{2}(\Delta \bar{J})^{2}-\frac{1}{2} C_{J \bar{J}}-(\Delta V)^{2} \\
\frac{\mathrm{d}}{\mathrm{d} \phi} C_{J \bar{J}} & =-C_{V J}-C_{V \bar{J}} .
\end{aligned}
$$

They are solved by 46

$$
\begin{aligned}
(\Delta V)^{2}(\phi) & =\frac{1}{2}\left(c_{3} e^{-2 \phi}+c_{4} e^{2 \phi}\right)-\frac{1}{4}\left(c_{1}+c_{2}\right) \\
(\Delta J)^{2}(\phi) & =\frac{1}{2}\left(c_{3} e^{-2 \phi}+c_{4} e^{2 \phi}\right)+\frac{1}{4}\left(3 c_{2}-c_{1}\right)-i\left(c_{5} e^{\phi}-c_{6} e^{-\phi}\right) \\
(\Delta \bar{J})^{2}(\phi) & =\frac{1}{2}\left(c_{3} e^{-2 \phi}+c_{4} e^{2 \phi}\right)+\frac{1}{4}\left(3 c_{2}-c_{1}\right)+i\left(c_{5} e^{\phi}-c_{6} e^{-\phi}\right) \\
C_{V J}(\phi) & =\frac{1}{2}\left(c_{3} e^{-2 \phi}-c_{4} e^{2 \phi}\right)+\frac{i}{2}\left(c_{5} e^{\phi}+c_{6} e^{-\phi}\right) \\
C_{V \bar{J}}(\phi) & =\frac{1}{2}\left(c_{3} e^{-2 \phi}-c_{4} e^{2 \phi}\right)-\frac{i}{2}\left(c_{5} e^{\phi}+c_{6} e^{-\phi}\right) \\
C_{J \bar{J}}(\phi) & =\frac{1}{2}\left(c_{3} e^{-2 \phi}+c_{4} e^{2 \phi}\right)+\frac{1}{4}\left(3 c_{1}-c_{2}\right)
\end{aligned}
$$

with independent integration constants $c_{1}, \ldots, c_{6}$. Reality conditions are already implemented for real $c_{I}$ since this implies $C_{V \bar{J}}=\overline{C_{V J}}$ and $(\Delta \bar{J})^{2}=\overline{(\Delta J)^{2}}$. Solutions can be chosen to saturate uncertainty relations in order to determine properties of dynamical coherent states. This analysis has been performed in [46, 10] in order to shed light on the possible asymmetries of volume fluctuations before and after the bounce. For large $H$, which is required for a massive universe, state properties such as $c_{3} / c_{4}$ and $c_{5} / c_{6}$ are extremely sensitive to initial values. Thus, from current knowledge of the classicality of the universe one can practically derive nothing about the precise fluctuations of the state before the big bang. As we will see below, these same parameters are also crucial for the

\footnotetext{
${ }^{7}$ Note that it is not the square root $\Delta \bar{J}$ for which reality conditions are imposed because they are based on $\left\langle\left(\hat{J}^{\dagger}\right)^{2}\right\rangle-\left\langle\hat{J}^{\dagger}\right\rangle^{2}=\overline{\left\langle(\hat{J})^{2}\right\rangle-\langle\hat{J}\rangle^{2}}$.
} 
behavior near the would-be bounce at $\phi=0$ of an interacting system. We emphasize the role of $H$ : a large $H$ keeps the bounce away from small volume and its strong quantum effects, but heightens the sensitivity to small changes in an initial quantum state. Small $H$ would reduce the sensitivity, but also the bounce volume and thus introduce more quantum effects in the evolution. When discussing interactions in what follows we will see further implications of a large value of $H$.

\subsection{Interactions}

We are now ready to introduce an interaction term via a potential $W(\phi)$ of the scalar, which destroys the exact solvability and implies quantum back-reaction. There are several difficulties, which complicate a complete analysis. All this has been discussed in [12], and we present a brief review here: First, $\phi$ in general will no longer serve as a global internal time, such that one can only study patches of solutions in which $\phi$ would be a monotonic function of a coordinate time. Especially since we have to address a question about long evolution times, several patches are in general required. Secondly, the $\phi$-Hamiltonian $p_{\phi}$ is now time dependent, as it is a function of $\phi$ through the potential. While

$$
-p_{\phi}= \pm H= \pm \frac{3}{4 \pi G}|p| \sqrt{\frac{c^{2}}{\gamma^{2}}-\frac{8 \pi G}{3}|p| W(\phi)}
$$

can still be used as a classical Hamiltonian, upon quantization solutions to the equation $-\hat{p}_{\phi} \psi=i \hbar \partial \psi / \partial \phi= \pm \hat{H} \psi$ will no longer provide exact solutions of the original constraint equation (3) which is a second order differential equation in $\phi$ : for solutions $\psi$ of the linear equation, we have $\hat{p}_{\phi}^{2} \psi= \pm \hat{p}_{\phi} \hat{H} \psi=\hat{H}^{2} \psi \pm\left[\hat{p}_{\phi}, \hat{H}\right] \psi \neq \hat{H}^{2} \psi$. Finally, there are now couplings between all the quantum variables, which requires a detailed analysis of effective equations.

For a sufficiently small and flat potential, all these difficulties can be evaded: (i) While there is no global time, a small flat potential allows long monotonic changes of $\phi$ which one can analyze in one patch. Different patches can be combined, but this requires the consideration of general states as initial semiclassical states in the first patch will change in each patch and possibly in the patching process. (ii) The commutator $\left[\hat{p}_{\phi}, \hat{H}\right]$ which determines the error one makes by formulating the initial second order equation (3) as a first order Schrödinger equation ([6]) is proportional to $\hbar W^{\prime}$ and thus small for a flat potential. Moreover, since it arises from a commutator, one may view this extra term as simply a quantization ambiguity, or correct it by adding an extra contribution to the Hamiltonian $\hat{H}$ used for the linear equation. (iii) Coupling terms can be handled by suitable approximations in effective equations. This will be the main focus here.

\subsubsection{Effective equations}

To analyze implications for the bounce, we have to change the classical Hamiltonian (22) in two ways: we use canonical variables for general $x$, taking into account different refinement 
schemes of loop quantum cosmology, and then introduce the holonomy variable $J$ and its complex conjugate. This gives (again dropping, for now, numerical factors)

$$
H=\sqrt{-\frac{1}{4}(J-\bar{J})^{2}-V^{3 /(1-x)} W(\phi)}=\frac{J-\bar{J}}{2 i}-i \frac{V^{3 /(1-x)}}{J-\bar{J}} W(\phi)+\cdots
$$

in an expansion by the potential. For this non-linear classical Hamiltonian, the quantum Hamiltonian depends on all quantum variables. It can be derived from a formal Taylor expansion of the expression $H\left(\langle\hat{V}\rangle+(\hat{V}-\langle\hat{V}\rangle),\langle\hat{J}\rangle+(\hat{J}-\langle\hat{J}\rangle),\left\langle\hat{J}^{\dagger}\right\rangle+\left(\hat{J}^{\dagger}-\left\langle\hat{J}^{\dagger}\right\rangle\right)\right)$ in $\hat{V}-\langle\hat{V}\rangle, \hat{J}-\langle\hat{J}\rangle$ and $\hat{J}^{\dagger}-\left\langle\hat{J}^{\dagger}\right\rangle$. From the result one can derive Hamiltonian equations of motion via Poisson brackets between expectation values and quantum variables, a formal procedure which can be seen as a shortcut to computing expectation values of commutators $\langle[\cdot, \hat{H}]\rangle$.

In our case, we obtain the quantum Hamiltonian

$$
\begin{aligned}
H_{Q}= & \frac{J-\bar{J}}{2 i}-i \frac{V^{3 /(1-x)}}{J-\bar{J}} W(\phi) \\
& -\frac{3}{2} i \frac{2+x}{(1-x)^{2}} \frac{V^{(1+2 x)(1-x)}}{J-\bar{J}}(\Delta V)^{2} W(\phi)+\frac{3 i}{1-x} \frac{V^{(2+x) /(1-x)}}{(J-\bar{J})^{2}}\left(C_{V J}-C_{V \bar{J}}\right) W(\phi) \\
& -i \frac{V^{3 /(1-x)}}{(J-\bar{J})^{3}}\left((\Delta J)^{2}-2 C_{J \bar{J}}+(\Delta \bar{J})^{2}\right) W(\phi)+\cdots
\end{aligned}
$$

where the omitted terms now also include higher moments. We will regard this truncation as our perturbative effective Hamiltonian, which is valid as long as higher moment terms are subdominant.

Due to the new coupling terms, equations of motion become lengthy and we do not present all of them here. Complete equations to the order used here and details of their derivation can be found in 12 for $x=0$. The main equation of interest here is that for $\langle\hat{V}\rangle$ because it is the one giving us information about the bounce. From the effective Hamiltonian, we find its equation of motion

$$
\begin{aligned}
\frac{\mathrm{d}\langle\hat{V}\rangle}{\mathrm{d} \phi}= & -\frac{\langle\hat{J}\rangle+\left\langle\hat{J}^{\dagger}\right\rangle}{2}+\frac{\langle\hat{J}\rangle+\left\langle\hat{J}^{\dagger}\right\rangle}{\left(\langle\hat{J}\rangle-\left\langle\hat{J}^{\dagger}\right\rangle\right)^{2}}\langle\hat{V}\rangle^{3 /(1-x)} W(\phi) \\
& +3 \frac{2+x}{(1-x)^{2}} \frac{\langle\hat{J}\rangle+\left\langle\hat{J}^{\dagger}\right\rangle}{\left(\langle\hat{J}\rangle-\left\langle\hat{J}^{\dagger}\right\rangle\right)^{2}}\langle\hat{V}\rangle^{(1+2 x) /(1-x)}(\Delta V)^{2} W(\phi) \\
& +3 \frac{\langle\hat{J}\rangle+\left\langle\hat{J}^{\dagger}\right\rangle}{\left(\langle\hat{J}\rangle-\left\langle\hat{J}^{\dagger}\right\rangle\right)^{4}}\langle\hat{V}\rangle^{3 /(1-x)}\left((\Delta J)^{2}+(\Delta \bar{J})^{2}-2 C_{J \bar{J}}\right) W(\phi) \\
& -\frac{6}{1-x} \frac{\left\langle\hat{J}^{\prime}\right\rangle+\left\langle\hat{J}^{\dagger}\right\rangle}{\left(\langle\hat{J}\rangle-\left\langle\hat{J}^{\dagger}\right\rangle\right)^{3}}\langle\hat{V}\rangle^{(2+x) /(1-x)}\left(C_{V J}-C_{V \bar{J}}\right) W(\phi) \\
& -\frac{2\langle\hat{V}\rangle^{3 /(1-x)}}{\left(\langle\hat{J}\rangle-\left\langle\hat{J}^{\dagger}\right\rangle\right)^{3}}\left((\Delta J)^{2}-(\Delta \bar{J})^{2}\right) W(\phi) \\
& +\frac{3}{1-x} \frac{\langle\hat{V}\rangle^{(2+x) /(1-x)}}{\left(\langle\hat{J}\rangle-\left\langle\hat{J}^{\dagger}\right\rangle\right)^{2}}\left(C_{V J}+C_{V \bar{J}}\right) W(\phi) .
\end{aligned}
$$


The evolution of the volume expectation value now depends on the behavior of the state via its moments.

For semiclassical states, the correction terms are certainly small, but we recall that the singularity problem is a question about long-term evolution. Then, the state may change considerably and our equation, corresponding to the last phase before the big bang is reached, has to be applied to a state whose moments can be large. Additional correction terms will arise from higher moments neglected so far, but we can use the equation to test whether the fluctuations have an effect, and how this can occur precisely. Moreover, our conclusions can be extended to all orders in the potential and in quantum variables, which allows for arbitrary states [52]. We are here not interested in a precise analysis, which would seem premature, but in understanding the role of quantum corrections. Thus, we assume them to be small and check the self-consistency of this assumption.

\subsubsection{Correlations}

Let us thus assume that our state has for some time evolved by the exact solutions of the solvable model, corrected only slightly by the classical potential term and quantum backreaction. We may thus use the solutions (9) and (10) for expectation values and (16)-(21) for fluctuations and covariances as zeroth order solutions to estimate the magnitude of quantum back-reaction. In particular, we look at where the free solutions would have their bounce, thus implying $\langle\hat{J}\rangle+\left\langle\hat{J}^{\dagger}\right\rangle \approx 0$. This already eliminates several of the terms in (25), in particular the classical interaction. However, the last two quantum correction terms remain, which further using $\langle\hat{V}\rangle \approx H \approx-\frac{1}{2} i\left(\langle\hat{J}\rangle-\left\langle\hat{J}^{\dagger}\right\rangle\right)$ gives

$$
\frac{\mathrm{d}\langle\hat{V}\rangle}{\mathrm{d} \phi} \approx-\frac{1}{H^{-3 x /(1-x)}}\left(\frac{1}{2} \operatorname{Im}(\Delta J(\phi))^{2}+\frac{3}{2(1-x)} \operatorname{Re} C_{V J}(\phi)\right) W(\phi) .
$$

This vanishes around $\phi \approx 0$ for background solutions satisfying $c_{3} \sim c_{4}, c_{5} \sim c_{6}$, which corresponds to unsqueezed states. For squeezed states, however, the time derivative of $\langle\hat{V}\rangle$ does not vanish for the interacting system at the bounce of the free solutions. The interacting system cannot bounce where the free system would bounce. Thus, quantum back-reaction does affect the bounce for squeezed states. Whether or not and where the bounce may happen depends on quantum properties of the state, not just on expectation values as in the free case. Even for free solutions, there is not much control over the key parameters $c_{3} / c_{4}$ and $c_{5} / c_{6}$ due to cosmic forgetfulness: it is precisely these parameters which determine the asymmetry of fluctuations discussed in [9, 10]. It will thus be even more complicated to constrain these parameters in an interacting system. To estimate the precise effect we have to know how squeezing develops for the interacting solutions; otherwise no reliable statement about the persistence of the bounce in the perturbed system can be made.

In addition to cosmic forgetfulness, we have to consider the dynamical form of quantum variables and their coupling to expectation values in interacting states. Notice that the quantum corrections in (25) split into two classes: the first three lines which include 
$\langle\hat{J}\rangle+\left\langle\hat{J}^{\dagger}\right\rangle$ as a factor and which can be subsumed as changing the classical potential to $W(\phi)\left(1+\epsilon_{1}\right)$ with relative fluctuations

$$
\epsilon_{1}=3 \frac{2+x}{(1-x)^{2}} \frac{(\Delta V)^{2}}{\langle\hat{V}\rangle^{2}}+3 \frac{\left.(\Delta J)^{2}-2 C_{J \bar{J}}+\Delta \bar{J}\right)^{2}}{\left(\langle\hat{J}\rangle-\left(\left\langle\hat{J}^{\dagger}\right\rangle\right)^{2}\right.}-\frac{6}{1-x} \frac{C_{V J}-C_{V \bar{J}}}{\langle\hat{V}\rangle\left(\langle\hat{J}\rangle-\left\langle\hat{J}^{\dagger}\right\rangle\right)},
$$

and the last two lines which are present even when $\langle\hat{J}\rangle+\left\langle\hat{J}^{\dagger}\right\rangle$ vanishes.

These last terms are of special interest because they remain in the analysis of the bounce which led to (26). To see whether they are generically zero or, if not, how large they can grow, we need the equations of motion for $C_{V J}$ and $(\Delta J)^{2}$. This requires more Poisson brackets than presented so far, which are listed in App. A as recalled from [12]. In these relations, moments of third order appear (with triple superscript indices) which we ignore in our approximation together with the terms of order $\hbar^{2}$ or higher.

With these relations and the quantum Hamiltonian (24), we obtain

$$
\begin{aligned}
\frac{\mathrm{d}}{\mathrm{d} \phi}\left(\operatorname{Re} C_{V J}\right)= & -\frac{1}{2}\left(\operatorname{Re}(\Delta J)^{2}+C_{J \bar{J}}+2(\Delta V)^{2}\right) \\
& +2 \frac{\langle\hat{V}\rangle^{3 /(1-x)}}{\left(\langle\hat{J}\rangle-\left\langle\hat{J}^{\dagger}\right\rangle\right)^{2}}\left(C_{J \bar{J}}+\frac{10-x}{1-x}(\Delta V)^{2}\right) W(\phi) \\
& +\frac{3}{2} \frac{2+x}{(1-x)^{2}}\langle\hat{V}\rangle^{(1+2 x) /(1-x)}(\Delta V)^{2} W(\phi) \\
& +\left(\frac{6 i}{1-x} \frac{\langle\hat{V}\rangle^{(2+x) /(1-x)}}{\langle\hat{J}\rangle-\left\langle\hat{J}^{\dagger}\right\rangle}-12 i \frac{\langle\hat{V}\rangle^{(4-x) /(1-x)}}{\left(\langle\hat{J}\rangle-\left\langle\hat{J}^{\dagger}\right\rangle\right)^{3}}\right) \operatorname{Im} C_{V J} W(\phi) \\
& -\frac{3}{1-x} \frac{\langle\hat{V}\rangle^{(2+x) /(1-x)}\left(\langle\hat{J}\rangle+\left\langle\hat{J}^{\dagger}\right\rangle\right)}{\left(\langle\hat{J}\rangle-\left\langle\hat{J}^{\dagger}\right\rangle\right)^{2}} \operatorname{Re} C_{V J} W(\phi) \\
& -i \frac{\langle\hat{V}\rangle^{3 /(1-x)}\left(\langle\hat{J}\rangle+\left\langle\hat{J}^{\dagger}\right\rangle\right)}{\left(\langle\hat{J}\rangle-\left\langle\hat{J}^{\dagger}\right\rangle\right)^{3}} \operatorname{Im}(\Delta J)^{2} W(\phi)
\end{aligned}
$$

and

$$
\begin{aligned}
\frac{\mathrm{d}}{\mathrm{d} \phi}\left(\operatorname{Im}(\Delta J)^{2}\right)= & -2 \operatorname{Im} C_{V J}+4\left(\frac{5+x}{1-x} \frac{\langle\hat{V}\rangle^{3 /(1-x)}}{\left(\langle\hat{J}\rangle-\left\langle\hat{J}^{\dagger}\right\rangle\right)^{2}}-\frac{3(2+x)}{2(1-x)^{2}}\langle\hat{V}\rangle^{(1+2 x) /(1-x))}\right) \operatorname{Im} C_{V J} W(\phi) \\
& -2 i\left(3 \frac{2+x}{(1-x)^{2}}-4 \frac{\langle\hat{V}\rangle^{2}}{\left(\langle\hat{J}\rangle-\left\langle\hat{J}^{\dagger}\right\rangle\right)^{2}}\right) \frac{\langle\hat{V}\rangle^{(1+2 x) /(1-x)}\left(\langle\hat{J}\rangle+\left\langle\hat{J}^{\dagger}\right\rangle\right)}{\langle\hat{J}\rangle-\left\langle\hat{J}^{\dagger}\right\rangle} \operatorname{Re} C_{V J} W(\phi) \\
& -8 i \frac{\langle\hat{V}\rangle^{(4-x) /(1-x)}}{\left(\langle\hat{J}\rangle-\left\langle\hat{J}^{\dagger}\right\rangle\right)^{3}}\left(2(\Delta V)^{2}+C_{J \bar{J}}\right) W(\phi) \\
& -2 i\left(\frac{3}{1-x}-4 \frac{\langle\hat{V}\rangle^{2}}{\left(\langle\hat{J}\rangle-\left\langle\hat{J}^{\dagger}\right\rangle\right)^{2}}\right) \frac{\langle\hat{V}\rangle^{(2+x) /(1-x)}}{\langle\hat{J}\rangle-\left\langle\hat{J}^{\dagger}\right\rangle} \operatorname{Re}(\Delta J)^{2} W(\phi) \\
& -\frac{6}{1-x} \frac{\langle\hat{V}\rangle^{(2+x) /(1-x)}\left(\langle\hat{J}\rangle+\left\langle\hat{J}^{\dagger}\right\rangle\right)}{\left(\langle\hat{J}\rangle-\left\langle\hat{J}^{\dagger}\right\rangle\right)^{2}} \operatorname{Im}(\Delta J)^{2} W(\phi) .
\end{aligned}
$$


Derivatives of the correlations by $\phi$ cannot vanish because they are determined by some of the fluctuations which, thanks to uncertainty relations, are non-zero. For instance, $(\Delta V)^{2}$ appears in both equations and drives the change of squeezing. It is multiplied by the small potential, but also by factors of the volume. If we consider relative fluctuations $(\Delta V)^{2} / V^{2}$ of volume, which are nearly constant in each expanding or contracting branch of the free solutions, the dominating pre-factors are $\langle\hat{V}\rangle^{(5-2 x) /(1-x)} /\left(\langle\hat{J}\rangle-\left\langle\hat{J}^{\dagger}\right\rangle\right)^{2}$ for $\operatorname{Re} C_{V J}$ and $\langle\hat{V}\rangle^{3(2-x) /(1-x)} /\left(\langle\hat{J}\rangle-\left\langle\hat{J}^{\dagger}\right\rangle\right)^{3}$ for $\operatorname{Im}(\Delta J)^{2}$. Thus, especially at large volume, but also at the bounce itself, these terms can contribute to a significant change in time of squeezing. For $x=-1 / 2$, for instance, they behave as $V^{4} / H^{2}$ and $V^{5} / H^{3}$, respectively. For the expanded Hamiltonian, on the other hand, only $V^{2} / H^{2} W \ll 1$ is required, which allows the pre-factors to be large. Moreover, since the arguments can be extended to all orders in powers of the potential [52], the condition on $W$ to be small can be relaxed. When small volume is reached, squeezing parameters will be important in Eq. (26).

Eq. (26) then shows that a large $H$ has a protective effect for the bounce for $x \neq 0$, which is strongest for the limiting case $x=-1 / 2$. As before, we thus notice that a large $H$, which implies a bounce at large volume, preserves classicality better than small $H$, which is more pronounced the larger $-x$ is. However, the influence of squeezing is always present and is required for a proper understanding of the bounce. Also this is weaker for $x=-1 / 2$ than for $x=0$, but remains potentially large. We will now analyze the same effect from the point of view of an effective Friedmann equation which, unlike (14), correctly captures state properties in the presence of interactions.

\subsubsection{Effective Friedmann equation}

Using the effective Hamiltonian, which is the truncation of the quantum Hamiltonian explicitly written out in (24), and the $\phi$-evolution equation (25) of $\langle\hat{V}\rangle$, we can derive an effective Friedmann equation as before in Sec. 2.2.1. There are now several additional terms especially due to quantum variables, whose explicit dynamics we have not determined. We will therefore keep them in a general form as relative fluctuations such as (27) and see how they affect the Friedmann equation.

We first sketch the procedure and non-trivial changes due to the potential for the expanded Hamiltonian linear in $W$, but then use also the quadratic terms shown in App. B in the final effective Friedmann equation because they are expected if $\rho^{2}$-corrections are present. In the effective Hamiltonian, it turns out that all relative quantum variables can be summarized by a single multiplicative correction to the classical potential:

$$
H_{\mathrm{eff}}=\frac{J-\bar{J}}{2 i}-i \frac{V^{3 /(1-x)}}{J-\bar{J}} W(\phi)\left(1+\delta_{1}\right)
$$

with

$$
\delta_{1}=\frac{3}{2} \frac{2+x}{(1-x)^{2}} \frac{(\Delta V)^{2}}{\langle\hat{V}\rangle^{2}}+\frac{(\Delta J)^{2}-2 C_{J \bar{J}}+(\Delta \bar{J})^{2}}{\left(\langle\hat{J}\rangle-\left(\left\langle\hat{J}^{\dagger}\right\rangle\right)^{2}\right)}-\frac{3}{1-x} \frac{C_{V J}-C_{V \bar{J}}}{\langle\hat{V}\rangle\left(\langle\hat{J}\rangle-\left\langle\hat{J}^{\dagger}\right\rangle\right)}
$$

which differs from $\epsilon_{1}$ in (27) by some coefficients. 
To express $\mathrm{d} V / \mathrm{d} \phi$ in a similar form, we have to distinguish between two different contributions from quantum variables. One of them is $\epsilon_{1}$, which, like $\delta_{1}$, just multiplies the potential. But there are additional contributions in the last two lines of (25) containing squeezing parameters, which are not of this form and which we write as 8

$$
\eta_{1}=-2 \frac{(\Delta J)^{2}-(\Delta \bar{J})^{2}}{\langle\hat{V}\rangle\left(\langle\hat{J}\rangle-\left\langle\hat{J}^{\dagger}\right\rangle\right)}+\frac{3}{1-x} \frac{C_{V J}+C_{V \bar{J}}}{\langle\hat{V}\rangle^{2}}
$$

We then have the evolution equation

$$
\frac{\mathrm{d} V}{\mathrm{~d} \phi}=-\frac{J+\bar{J}}{2}+\frac{J+\bar{J}}{(J-\bar{J})^{2}} V^{3 /(1-x)} W(\phi)\left(1+\epsilon_{1}\right)+\frac{V^{(4-x) /(1-x)}}{(J-\bar{J})^{2}} \eta_{1} W(\phi) .
$$

With all numerical factors reinstated, we have

$$
H_{\mathrm{eff}}=2 \sqrt{\frac{4 \pi G}{3}}(1-x)\left(\frac{J-\bar{J}}{2 i}-i \frac{8 \pi G}{3}\left(\frac{8 \pi \gamma G f_{0}(1-x) V}{3}\right)^{3 /(1-x)} \frac{W(\phi)}{J-\bar{J}}\left(1+\delta_{1}\right)\right)=p_{\phi}
$$

which provides a quadratic equation for $J-\bar{J}$ in terms of $p_{\phi}$. Its perturbative solution in $W$ and fluctuations is

$$
2 i(J-\bar{J})=\sqrt{\frac{3}{4 \pi G}} \frac{p_{\phi}}{2(1-x)}\left(1+a^{6} \frac{W(\phi)}{p_{\phi}^{2}}\left(1+\delta_{1}\right)\right)
$$

and, via (13), provides

$$
\frac{J+\bar{J}}{2 V+\hbar}= \pm \sqrt{1-\frac{8 \pi \gamma^{2} G f_{0}^{2}}{3} a^{2+4 x} \rho+\sigma+O\left(\left(a^{6} W p_{\phi}^{-2}\right)^{2}\right)}
$$

with the energy density $\rho=\frac{1}{2} a^{-6} p_{\phi}^{2}+W(\phi)$ of the interacting scalar.

The evolution equation (32) now is to be squared for the effective Friedmann equation after replacing $J+\bar{J}$. Here, terms quadratic in the potential become important, and so we should include them in the quantum Hamiltonian expanded to second order in the potential. The complete expressions are provided in App. B, which, with all numerical

\footnotetext{
${ }^{8}$ There is some arbitrariness in what one considers as relative fluctuations. Had we used $\langle\hat{J}\rangle+\left\langle\hat{J}^{\dagger}\right\rangle$ instead of what appears in $\eta_{1}$, then there would be a total factor of $\langle\hat{J}\rangle+\left\langle\hat{J}^{\dagger}\right\rangle$ and $\eta_{1}$ could be subsumed in $\epsilon_{1}$. It might, in fact, appear more natural to use, e.g., $\left(C_{V J}+C_{V \bar{J}}\right) /\langle\hat{V}\rangle\left(\langle\hat{J}\rangle+\left\langle\hat{J}^{\dagger}\right\rangle\right)$ instead of $\left(C_{V J}+C_{V \bar{J}}\right) /\langle\hat{V}\rangle^{2}$. However, it is dangerous to do that because one would divide by a quantity, $\langle\hat{J}\rangle+\left\langle\hat{J}^{\dagger}\right\rangle$, which then vanishes at the bounce. The quantity $\eta_{1}$, on the other hand, simply absorbs factors which are already present in the equation or which do not vanish, and does so in a way which makes the final quantity dimensionless. Moreover, in each expanding or contracting branch $\langle\hat{V}\rangle$ behaves approximately as $\langle\hat{J}\rangle+\left\langle\hat{J}^{\dagger}\right\rangle$ but does not become zero. All relative quantum variables defined here are nearly constant for the free solutions in each branch, although they can change during the free bounce.
} 
factors reinstated, provides the effective Friedmann equation

$$
\begin{aligned}
& \left(\frac{\dot{a}}{a}\right)^{2}=\frac{8 \pi G}{3}\left(\rho+\epsilon_{1} W(\phi)-2 \xi_{2} a^{6} \frac{W^{2}}{p_{\phi}^{2}}\right) \\
& \times\left(1-\frac{8 \pi G}{3} \gamma^{2} f_{0}^{2} a^{2+4 x}\left(\rho+W(\phi) \delta_{1}+\frac{\xi_{1}}{2} a^{6} \frac{W^{2}}{p_{\phi}^{2}}\right)+\sigma\right) \\
& \pm \frac{4 \pi G}{3} W(\phi) \sqrt{1-\frac{8 \pi G}{3} \gamma^{2} f_{0}^{2} a^{2+4 x}\left(\rho+W(\phi) \delta_{1}+\frac{\xi_{1}}{2} a^{6} \frac{W^{2}}{p_{\phi}^{2}}\right)+\sigma} \\
& \times\left(\eta_{1}+\xi_{3} \frac{a^{6} W(\phi)}{p_{\phi}^{2}}\right) \\
& +\frac{4 \pi G}{3} \frac{a^{6} W(\phi)^{2}}{p_{\phi}^{2}} \eta_{1}^{2} \text {. }
\end{aligned}
$$

The \pm corresponds to the sign of $J+\bar{J}$ as it arises when the square root is taken. Coefficients denoted by Greek letters are relative quantum variables defined in the appendix or earlier in this section. From this equation, it does not follow in an obvious manner that there should be a bounce. As it stands, this equation may have no solution for $\dot{a}$ - or several ones. Also here, we clearly see the additional contribution by the squeezing term which is essential to decide about the bounce, i.e. to find out where, if at all, $\dot{a}=0$. If there would be no squeezing, i.e. $\eta_{1}=0=\eta_{2}$, then the scale factor would bounce for an energy density near the critical value of the free model, only slightly corrected by $\epsilon_{1}, \delta_{1}$ and $\sigma$.

Instead of using the momentum and potential of $\phi$, we can express the effective Friedmann equation in terms of energy density and pressure $P$ :

$$
\begin{aligned}
\left(\frac{\dot{a}}{a}\right)^{2}=\frac{8 \pi G}{3} & \left(\rho+\frac{1}{2} \epsilon_{1}(\rho-P)-\xi_{2} \frac{(\rho-P)^{2}}{\rho+P}\right) \\
& \times\left(1-\frac{1}{\rho_{\text {crit }}}\left(\rho+\frac{\delta_{1}}{2}(\rho-P)+\frac{\xi_{1}}{4} \frac{(\rho-P)^{2}}{\rho+P}\right)+\sigma\right) \\
\pm & \frac{2 \pi G}{3}(\rho-P) \sqrt{1-\frac{1}{\rho_{\text {crit }}}\left(\rho+\frac{\delta_{1}}{2}(\rho-P)+\frac{\xi_{1}}{4} \frac{(\rho-P)^{2}}{\rho+P}\right)+\sigma} \\
& \times\left(\eta_{1}+\frac{\xi_{3}}{2} \frac{(\rho-P)^{2}}{\rho+P}\right) \\
+ & \frac{2 \pi G}{3} \frac{(\rho-P)^{2}}{\rho+P} \eta_{1}^{2}
\end{aligned}
$$

where $\rho_{\text {crit }}=3 / 8 \pi G \gamma^{2} f_{0}^{2} a^{2+4 x}$ is the same as in the free case. However, $\rho=\rho_{\text {crit }}$ would give a bounce only in special cases. 


\section{Conclusions}

We have derived effective equations for isotropic models in loop quantum cosmology sourced by self-interacting or massive matter. In contrast to models studied so far in this context, this introduces quantum back-reaction which implies that the changing shape of a quantum state influences the motion of its expectation values. This effect, while initially negligible if one starts with a semiclassical state, is important for the long-term evolution of cosmology. The question of whether the classical singularity is replaced by a bounce can be reliably addressed only if quantum back-reaction is understood. Also the discussion of cyclic scenarios for the universe, or any situation of long evolution times, requires these terms to be taken into account.

We have derived perturbative effective equations which include quantum back-reaction terms, based on the availability of the exactly solvable model of [8] as free system. Compared to an effective Friedmann equation, which was previously available for models sourced by a free, massless scalar, there are new terms when the scalar has a potential. Phenomenologically, this implies that the effective Friedmann equation depends on the pressure of matter and not just on its energy density as in the classical or free quantum case. Compared to the free equation additional terms, which are independent of $f_{0}$, do not arise from discreteness or repulsive gravity but from genuine quantum back-reaction. These terms do grow in long-term evolution and are especially important where the free system would bounce. We have proven that there is still a bounce provided that the state at small volume is uncorrelated, which presents a substantial generalization of what has been available so far. However, we have also demonstrated that the bounce is affected by quantum backreaction of correlations, and much more work is required to see how generically a bounce is realized in this case. The now available equations, including those for moments, are suitable for detailed numerical studies of the whole parameter space. However, the parameters relevant for the bounce are exactly the ones subject to cosmic forgetfulness [9, 10], such that a firm and robust statement about the bounce may be difficult to achieve.

Moreover, our quantum back-reaction equations show that a generic state does develop strong quantum properties, especially correlations, which are important for the space-time picture at small volume and once a high-curvature region is traversed. This implies that space-time does not stay as classical as detailed investigations of the free model suggested; quantum geometry in general does become important. It makes itself noticeable not just in repulsive forces but also in genuine quantum variables such as fluctuations and correlations. While quantum evolution remains non-singular at a basic level of the whole wave function, in general there may not be a smooth geometrical picture such as a simple bounce of only the classical volume where genuine quantum variables would play no role.

For isotropic models, there are several places where a large matter content $H$ helps to reduce the effects of quantum physics, thus making the evolution appear more classical. (But note that the precise rate of reduction depends on the refinement scheme of loop quantum gravity which is only effectively included in isotropic models.) There are, how-

ever, two important properties which prevent one from using this for a general physical conclusion to the extent that effects of quantum variables may be weak. First, already in 
isotropic models, a large matter content enhances the sensitivity to quantum properties of an initial state [10] which, in long-term evolution, again makes quantum effects play a large role. Secondly, the scale of quantum effects is determined by the total matter content in the universe only for homogeneous models, while inhomogeneous situations are based on more local variables [17]. Those variables would generically be much smaller than the total matter content unless the spatial size of the universe is very tiny and comparable to the Planck volume. In either case, one cannot escape strong quantum effects when the small-volume behavior of cosmological models is considered. Thus, the behavior for small $H$ is also of vital interest even though an isotropic model for a large universe would require large $H$. Similarly to a single massive partile in quantum mechanics, which does not show strong quantum properties in contrast to the microscopic dynamics of its elementary constituents, quantum aspects of a large isotropic universe seem suppressed by its large matter content. However, an isotropic model does not fully capture the microscopic quantum gravitational dynamics, and it is this dynamics which must be shown to be non-singular. Within isotropic models one can obtain indications by looking at their properties also for small $H$. While the basic removal of singularities based on an extendability of wave functions has been shown also for inhomogeneous systems of spherical symmetry [45], which thus captures microscopic dynamics, this may in general not correspond to a smooth bounce.

Acknowledgements: This work was supported in part by NSF grant PHY0653127.

\section{Appendices}

\section{A Some useful Poisson brackets}

For equations of motion of quantum variables, we are using Poisson brackets

$$
\begin{aligned}
\left\{C_{V J},\langle\hat{V}\rangle\right\} & =i C_{V J}, \quad\left\{C_{V J},\langle\hat{J}\rangle\right\}=-i(\Delta J)^{2} \quad, \quad\left\{C_{V J},\left\langle\hat{J}^{\dagger}\right\rangle\right\}=i C_{J \bar{J}}+2 i(\Delta V)^{2} \\
\left\{C_{V J},(\Delta V)^{2}\right\} & =2 i G^{V V J}+2 i\langle\hat{J}\rangle(\Delta V)^{2}-\frac{1}{6} i \hbar^{2}\langle\hat{J}\rangle \\
\left\{C_{V J}, C_{V \bar{J}}\right\} & =4 i G^{V V V}+6 i\left(\langle\hat{V}\rangle+\frac{1}{2} \hbar\right)(\Delta V)^{2}-i\langle\hat{J}\rangle C_{V \bar{J}}-i\left\langle\hat{J}^{\dagger}\right\rangle C_{V J}+\frac{1}{2} i \hbar^{2}\langle\hat{V}\rangle+\frac{1}{4} i \hbar^{3} \\
\left\{C_{V J},(\Delta J)^{2}\right\} & =-2 i G^{J J}-2 i\langle\hat{J}\rangle(\Delta J)^{2} \\
\left\{C_{V J}, C_{J \bar{J}}\right\} & =2 i G^{V V J}+2 i\left(\langle\hat{V}\rangle+\frac{1}{2} \hbar\right) C_{V J}-i\langle\hat{J}\rangle C_{J \bar{J}}+i\left\langle\hat{J}^{\dagger}\right\rangle(\Delta J)^{2}-\frac{1}{6} i \hbar^{2}\langle\hat{J}\rangle \\
\left\{C_{V J},(\Delta \bar{J})^{2}\right\} & =6 i G^{V V \bar{J}}+8 i\left(\langle\hat{V}\rangle+\frac{1}{2} \hbar\right) C_{V \bar{J}}-2 i\langle\hat{J}\rangle(\Delta \bar{J})^{2}-i \hbar^{2}\left\langle\hat{J}^{\dagger}\right\rangle
\end{aligned}
$$

for $C_{V J}$ and

$$
\begin{aligned}
\left\{(\Delta J)^{2},\langle\hat{V}\rangle\right\} & =2 i(\Delta J)^{2} \quad, \quad\left\{(\Delta J)^{2},\langle\hat{J}\rangle\right\}=0 \quad, \quad\left\{(\Delta J)^{2},\left\langle\hat{J}^{\dagger}\right\rangle\right\}=4 i C_{V J} \\
\left\{(\Delta J)^{2},(\Delta V)^{2}\right\} & =4 i G^{V J}+4 i\langle\hat{J}\rangle C_{V J}
\end{aligned}
$$




$$
\begin{aligned}
\left\{(\Delta J)^{2}, C_{V J}\right\}= & 2 i G^{J J J}+2 i\langle\hat{J}\rangle(\Delta J)^{2} \\
\left\{(\Delta J)^{2}, C_{V \bar{J}}\right\}= & 6 i G^{V V J}+8 i\left(\langle\hat{V}\rangle+\frac{1}{2} \hbar\right) C_{V J}-2 i\left\langle\hat{J}^{\dagger}\right\rangle(\Delta J)^{2}-i \hbar^{2}\langle\hat{J}\rangle \\
\left\{(\Delta J)^{2}, C_{J \bar{J}}\right\}= & 4 i G^{V J J}+4 i\left(\langle\hat{V}\rangle+\frac{1}{2} \hbar\right)(\Delta J)^{2} \\
\left\{(\Delta J)^{2},(\Delta \bar{J})^{2}\right\}= & 8 i G^{V V V}+16 i\left(\langle\hat{V}\rangle+\frac{1}{2} \hbar\right)(\Delta V)^{2}+8 i\left(\langle\hat{V}\rangle+\frac{1}{2} \hbar\right) C_{J \bar{J}}-8 i\langle\hat{J}\rangle C_{V \bar{J}} \\
& -8 i\left\langle\hat{J}^{\dagger}\right\rangle C_{V J}+4 i \hbar^{2}\langle\hat{V}\rangle+2 i \hbar^{3}
\end{aligned}
$$

for $(\Delta J)^{2}$. They can be derived from expectation values of quantum commutators as discussed in more detail in [12].

\section{B Expansion to second order in the potential}

To second order in the potential, we have the classical Hamiltonian

$$
\begin{aligned}
H & =\sqrt{-\frac{1}{4}(J-\bar{J})^{2}-V^{3 /(1-x)} W(\phi)} \\
& =\frac{J-\bar{J}}{2 i}-i \frac{V^{3 /(1-x)}}{(J-\bar{J})^{2}} W+i \frac{V^{6 /(1-x)}}{(J-\bar{J})^{3}} W^{2}+\cdots
\end{aligned}
$$

which implies the quantum Hamiltonian

$$
\begin{aligned}
H_{Q}= & H_{Q}^{\operatorname{lin}}+W^{2}\left(i \frac{V^{6 /(1-x)}}{(J-\bar{J})^{3}}+3 i \frac{5+x}{(1-x)^{2}} \frac{V^{2(2+x) /(1-x)}}{(J-\bar{J})^{3}}(\Delta V)^{2}\right. \\
& \left.-\frac{18 i}{1-x} \frac{V^{(5+x)(1-x)}}{(J-\bar{J})^{4}}\left(C_{V J}-C_{V \bar{J}}\right)+6 i \frac{V^{6 /(1-x)}}{(J-\bar{J})^{5}}\left((\Delta J)^{2}-2 C_{J \bar{J}}+(\Delta \bar{J})^{2}\right)\right) \\
= & \frac{J-\bar{J}}{2 i}-i \frac{V^{3 /(1-x)}}{J-\bar{J}}\left(1+\delta_{1}\right) W+i \frac{V^{6 /(1-x)}}{(J-\bar{J})^{3}}\left(1+\delta_{2}\right) W^{2}
\end{aligned}
$$

where "lin" denotes terms linear in $W$ already presented in the main text. In the last line, we have defined

$$
\delta_{2}:=3 \frac{5+x}{(1-x)^{2}} \frac{(\Delta V)^{2}}{\langle\hat{V}\rangle^{2}}+6 \frac{(\Delta J)^{2}-2 C_{J \bar{J}}+(\Delta \bar{J})^{2}}{\left(\langle\hat{J}\rangle-\left\langle\hat{J}^{\dagger}\right\rangle\right)^{2}}-\frac{18}{1-x} \frac{C_{V J}-C_{V \bar{J}}}{\langle\hat{V}\rangle\left(\langle\hat{J}\rangle-\left\langle\hat{J}^{\dagger}\right\rangle\right)}
$$

in addition to $\delta_{1}$ in (30).

From this Hamiltonian, we derive the equation of motion

$$
\begin{aligned}
\frac{\mathrm{d} V}{\mathrm{~d} \phi}= & -\frac{J+\bar{J}}{2}+\frac{J+\bar{J}}{(J-\bar{J})^{2}} V^{3 /(1-x)}\left(1+\epsilon_{1}\right) W-3 \frac{J+\bar{J}}{(J-\bar{J})^{4}} V^{6 /(1-x)}\left(1+\epsilon_{2}\right) W^{2} \\
& +\frac{V^{(4-x) /(1-x)}}{(J-\bar{J})^{2}} \eta_{1} W-3 \frac{V^{(7-x) /(1-x)}}{(J-\bar{J})^{4}} \eta_{2} W^{2}
\end{aligned}
$$


with

$$
\begin{aligned}
\epsilon_{2} & :=3 \frac{5+x}{(1-x)^{2}} \frac{(\Delta V)^{2}}{\langle\hat{V}\rangle^{2}}+10 \frac{\left((\Delta J)^{2}-2 C_{J \bar{J}}+\Delta \bar{J}\right)^{2}}{\left(\langle\hat{J}\rangle-\left\langle\hat{J}^{\dagger}\right\rangle\right)^{2}}-\frac{24}{1-x} \frac{C_{V J}-C_{V \bar{J}}}{\langle\hat{V}\rangle\left(\langle\hat{J}\rangle-\left\langle\hat{J}^{\dagger}\right\rangle\right)} \\
\eta_{2} & :=-4 \frac{(\Delta J)^{2}-(\Delta \bar{J})^{2}}{\langle\hat{V}\rangle\left(\langle\hat{J}\rangle-\left\langle\hat{J}^{\dagger}\right\rangle\right)}+\frac{6}{1-x} \frac{C_{V J}+C_{V \bar{J}}}{\langle\hat{V}\rangle^{2}}
\end{aligned}
$$

in addition to $\epsilon_{1}$ in (27) and $\eta_{1}$ in (31).

The equality $H_{\text {eff }}=p_{\phi}$ now implies a fourth-order polynomial for $J-\bar{J}$. To quadratic order in $W$, its perturbative solution is

$$
\frac{J-\bar{J}}{2 i}=p_{\phi}\left(1+\frac{1}{2} \frac{V^{3 /(1-x)}}{p_{\phi}^{2}}\left(1+\delta_{1}\right) W-\frac{1}{4} \frac{V^{6 /(1-x)}}{p_{\phi}^{4}}\left(1+2 \delta_{1}+\delta_{1}^{2}-\delta_{2} / 2\right) W^{2}\right) .
$$

Inserting this into (38) and using (13), we obtain

$$
\begin{aligned}
\frac{1}{V^{2}}\left(\frac{\mathrm{d} V}{\mathrm{~d} \phi}\right)^{2}= & \left(1-\frac{p_{\phi}^{2}}{V^{2}}-V^{(1+2 x) /(1-x)}\left(1+\delta_{1}\right) W-\frac{1}{4} \frac{V^{2(2+x) /(1-x)}}{p_{\phi}^{2}} \xi_{1} W^{2}+\sigma\right) \\
& \times\left(1+\frac{V^{3 /(1-x)}}{p_{\phi}^{2}}\left(1+\epsilon_{1}\right) W-\frac{V^{6 /(1-x)}}{p_{\phi}^{4}} \xi_{2} W^{2}\right) \\
\pm & \sqrt{2} \sqrt{1-\frac{p_{\phi}^{2}}{V^{2}}-V^{(1+2 x) /(1-x)}\left(1+\delta_{1}\right) W-\frac{1}{4} \frac{V^{2(2+x) /(1-x)}}{p_{\phi}^{2}} \xi_{1} W^{2}+\sigma} \\
& \times \frac{V^{3 /(1-x)} W}{p_{\phi}^{2}}\left(\eta_{1}+\frac{1}{2} \xi_{3} \frac{V^{3 /(1-x)} W}{p_{\phi}^{2}}\right) \\
+ & \frac{V^{6 /(1-x)}}{4 p_{\phi}^{4}} \eta_{1}^{2} W^{2}
\end{aligned}
$$

with

$$
\begin{aligned}
& \xi_{1}:=1+2 \delta_{1}+\delta_{2}+\delta_{1}^{2}, \\
& \xi_{2}:=\frac{1}{2} \epsilon_{1}+\delta_{1}-\frac{3}{4} \epsilon_{2}+\epsilon_{1} \delta_{2}-\frac{1}{4} \epsilon_{1}^{2}, \\
& \xi_{3}:=-\eta_{1}-\frac{3}{2} \eta_{2}+\eta_{1} \epsilon_{1}-2 \eta_{1} \delta_{1} .
\end{aligned}
$$

Reinstating numerical factors, this provides the effective Friedmann equation (33).

\section{References}

[1] M. Bojowald, Loop Quantum Cosmology,

ing Rev. Relativity 8 (2005) 11, gr-qc/0601085], http://relativity.livingreviews.org/Articles/lrr-2005-11/ 
[2] M. Bojowald, Absence of a Singularity in Loop Quantum Cosmology, Phys. Rev. Lett. 86 (2001) 5227-5230, gr-qc/0102069]

[3] M. Bojowald, Isotropic Loop Quantum Cosmology, Class. Quantum Grav. 19 (2002) 2717-2741, gr-qc/0202077

[4] A. Ashtekar, M. Bojowald, and J. Lewandowski, Mathematical structure of loop quantum cosmology, Adv. Theor. Math. Phys. 7 (2003) 233-268, gr-qc/0304074

[5] M. Bojowald, Singularities and Quantum Gravity, AIP Conf. Proc. 910 (2007) 294333, gr-qc/0702144], In: Proceedings of the XIIth Brazilian School on Cosmology and Gravitation

[6] A. Ashtekar, T. Pawlowski, and P. Singh, Quantum Nature of the Big Bang, Phys. Rev. Lett. 96 (2006) 141301, gr-qc/0602086]

[7] A. Ashtekar, T. Pawlowski, and P. Singh, Quantum Nature of the Big Bang: An Analytical and Numerical Investigation, Phys. Rev. D 73 (2006) 124038, gr-qc/0604013

[8] M. Bojowald, Large scale effective theory for cosmological bounces, Phys. Rev. D 75 (2007) 081301(R), gr-qc/0608100

[9] M. Bojowald, What happened before the big bang?, Nature Physics 3 (2007) 523-525

[10] M. Bojowald, Harmonic cosmology: How much can we know about a universe before the big bang?, arXiv:0710.4919

[11] A. Corichi and P. Singh, Quantum bounce and cosmic recall, arXiv:0710.4543

[12] M. Bojowald, H. Hernández, and A. Skirzewski, Effective equations for isotropic quantum cosmology including matter, Phys. Rev. D 76 (2007) 063511, arXiv:0706.1057]

[13] M. Bojowald and H. A. Kastrup, Symmetry Reduction for Quantized Diffeomorphism Invariant Theories of Connections, Class. Quantum Grav. 17 (2000) 3009-3043, hep-th/9907042

[14] M. Bojowald, H. H. Hernández, and H. A. Morales-Técotl, Perturbative degrees of freedom in loop quantum gravity: Anisotropies, Class. Quantum Grav. 23 (2006) 3491-3516, gr-qc/0511058]

[15] J. Engle, Quantum field theory and its symmetry reduction, Class. Quant. Grav. 23 (2006) 2861-2893, gr-qc/0511107]

[16] T. Koslowski, Reduction of a Quantum Theory, gr-qc/0612138

[17] M. Bojowald, Loop quantum cosmology and inhomogeneities, Gen. Rel. Grav. 38 (2006) 1771-1795, gr-qc/0609034 
[18] J. Engle, Relating loop quantum cosmology to loop quantum gravity: symmetric sectors and embeddings, Class. Quantum Grav. 24 (2007) 5777-5802, gr-qc/0701132

[19] T. Koslowski, A Cosmological Sector in Loop Quantum Gravity, arXiv:0711.1098

[20] N. Weiss, Constraints on Hamiltonian lattice formulations of field theories in an expanding universe, Phys. Rev. D 32 (1985) 3228-3232

[21] W. Unruh, Time, gravity, and quantum mechanics, In: Time's arrows today, Ed.: S. F. Savitt, pp. 23-94, gr-qc/9312027

[22] T. Jacobson, Trans-Planckian redshifts and the substance of the space-time river, hep-th/0001085

[23] R. Doldán, R. Gambini, and P. Mora, Quantum mechanics for totally constrained dynamical systems and evolving Hilbert spaces, Int. J. Theor. Phys. 35 (1996) 2057, hep-th/9404169

[24] M. Bojowald, The dark side of a patchwork universe, Gen. Rel. Grav. (2007) to appear, arXiv:0705.4398]

[25] A. Ashtekar, T. Pawlowski, and P. Singh, Quantum Nature of the Big Bang: Improved dynamics, Phys. Rev. D 74 (2006) 084003, gr-qc/0607039

[26] M. Bojowald, D. Cartin, and G. Khanna, Lattice refining loop quantum cosmology, anisotropic models and stability, Phys. Rev. D 76 (2007) 064018, arXiv:0704.1137

[27] W. Nelson and M. Sakellariadou, Lattice Refining LQC and the Matter Hamiltonian, Phys. Rev. D 76 (2007) 104003, arXiv:0707.0588

[28] W. Nelson and M. Sakellariadou, Lattice Refining Loop Quantum Cosmology and Inflation, Phys. Rev. D 76 (2007) 044015, arXiv:0706.0179]

[29] M. Bojowald and G. Hossain, Cosmological vector modes and quantum gravity effects, Class. Quantum Grav. 24 (2007) 4801-4816, arXiv:0709.0872

[30] M. Bojowald and G. Hossain, Quantum gravity corrections to gravitational wave dispersion, Phys. Rev. D 77 (2008) 023508, arXiv:0709.2365]

[31] K. Vandersloot, On the Hamiltonian Constraint of Loop Quantum Cosmology, Phys. Rev. D 71 (2005) 103506, gr-qc/0502082

[32] M. Bojowald, Inverse Scale Factor in Isotropic Quantum Geometry, Phys. Rev. D 64 (2001) 084018, gr-qc/0105067

[33] T. Thiemann, QSD V: Quantum Gravity as the Natural Regulator of Matter Quantum Field Theories, Class. Quantum Grav. 15 (1998) 1281-1314, gr-qc/9705019 
[34] M. Bojowald, Quantization ambiguities in isotropic quantum geometry, Class. Quantum Grav. 19 (2002) 5113-5130, gr-qc/0206053]

[35] M. Bojowald, Loop Quantum Cosmology: Recent Progress, Pramana 63 (2004) 765776, gr-qc/0402053 In: Proceedings of the International Conference on Gravitation and Cosmology (ICGC 2004), Cochin, India

[36] M. Bojowald, Loop Quantum Cosmology IV: Discrete Time Evolution, Class. Quantum Grav. 18 (2001) 1071-1088, gr-qc/0008053]

[37] J. Brunnemann and T. Thiemann, Unboundedness of Triad-Like Operators in Loop Quantum Gravity, Class. Quantum Grav. 23 (2006) 1429-1483, grr-qc/0505033|

[38] M. Bojowald, Degenerate Configurations, Singularities and the Non-Abelian Nature of Loop Quantum Gravity, Class. Quantum Grav. 23 (2006) 987-1008, gr-qc/0508118

[39] T. Thiemann, Quantum Spin Dynamics (QSD), Class. Quantum Grav. 15 (1998) 839-873, gr-qc/9606089

[40] T. Thiemann, Quantum Spin Dynamics (QSD) II: The Kernel of the Wheeler-DeWitt Constraint Operator, Class. Quantum Grav. 15 (1998) 875-905, gr-qc/9606090]

[41] S. Sabharwal and G. Khanna, Numerical solutions to lattice-refined models in loop quantum cosmology, arXiv:0711.2086

[42] M. Bojowald, The Semiclassical Limit of Loop Quantum Cosmology, Class. Quantum Grav. 18 (2001) L109-L116, gr-qc/0105113]

[43] M. Bojowald, Homogeneous loop quantum cosmology, Class. Quantum Grav. 20 (2003) 2595-2615, gr-qc/0303073

[44] M. Bojowald, G. Date, and K. Vandersloot, Homogeneous loop quantum cosmology: The role of the spin connection, Class. Quantum Grav. 21 (2004) 1253-1278, gr-qc/0311004

[45] M. Bojowald, Non-singular black holes and degrees of freedom in quantum gravity, Phys. Rev. Lett. 95 (2005) 061301, [gr-qc/0506128]

[46] M. Bojowald, Dynamical coherent states and physical solutions of quantum cosmological bounces, Phys. Rev. D 75 (2007) 123512, gr-qc/0703144

[47] M. Bojowald and A. Skirzewski, Effective Equations of Motion for Quantum Systems, Rev. Math. Phys. 18 (2006) 713-745, math-ph/0511043

[48] M. Bojowald and A. Skirzewski, Quantum Gravity and Higher Curvature Actions, Int. J. Geom. Meth. Mod. Phys. 4 (2007) 25-52, [hep-th/0606232], In: Proceedings of "Current Mathematical Topics in Gravitation and Cosmology" (42nd Karpacz Winter School of Theoretical Physics), Ed. Borowiec, A. and Francaviglia, M. 
[49] P. Singh, Loop cosmological dynamics and dualities with Randall-Sundrum braneworlds, Phys. Rev. D 73 (2006) 063508, gr-qc/0603043]

[50] G. Date and G. M. Hossain, Effective Hamiltonian for Isotropic Loop Quantum Cosmology, Class. Quantum Grav. 21 (2004) 4941-4953, gr-qc/0407073]

[51] G. Date and G. M. Hossain, Genericity of Big Bounce in isotropic loop quantum cosmology, Phys. Rev. Lett. 94 (2005) 011302, gr-qc/0407074

[52] M. Bojowald, in preparation 